\title{
The Health-Seeking Behavior among Malaysian Adults in Urban and Rural Areas Who Reported Sickness: Findings from the National Health and Morbidity Survey (NHMS) 2019
}

\author{
Sarah Nurain Mohd Noh ${ }^{1,2, *,+}$, Suhana Jawahir ${ }^{1,2,+} \mathbb{D}$, Yeung R'ong Tan ${ }^{1,2} \mathbb{D}$, Iqbal Ab Rahim ${ }^{1,2} \mathbb{D}$ \\ and Ee Hong Tan 1,3 \\ 1 Centre for Health Equity Research, Institute for Health Systems Research, Ministry of Health Malaysia \\ Shah Alam 40170, Malaysia; suhana.j@moh.gov.my (S.J.); dr.tanyr@moh.gov.my (Y.R.T.); \\ fathullah@moh.gov.my (I.A.R.); jdreehong@moh.gov.my (E.H.T.) \\ 2 National Institutes of Health, Ministry of Health Malaysia, Shah Alam 40170, Malaysia \\ 3 Melaka State Health Department, Ministry of Health Malaysia, Ayer Keroh 75450, Malaysia \\ * Correspondence: sarahnurain.mn@moh.gov.my; Tel.: +60-333627500 \\ + These authors contributed equally to this work.
}

Citation: Mohd Noh, S.N.; Jawahir, S.; Tan, Y.R.; Ab Rahim, I.;

Tan, E.H. The Health-Seeking Behavior among Malaysian Adults in Urban and Rural Areas Who Reported Sickness: Findings from the National Health and Morbidity Survey (NHMS) 2019. Int. J. Environ. Res. Public Health 2022, 19, 3193. https://doi.org/10.3390/ ijerph19063193

Academic Editor: Meng-Chih Lee

Received: 6 December 2021 Accepted: 17 February 2022 Published: 8 March 2022

Publisher's Note: MDPI stays neutral with regard to jurisdictional claims in published maps and institutional affiliations.

Copyright: (c) 2022 by the authors Licensee MDPI, Basel, Switzerland. This article is an open access article distributed under the terms and conditions of the Creative Commons Attribution (CC BY) license (https:// creativecommons.org/licenses/by/ $4.0 /)$.
Abstract: Understanding care-seeking behavior among urban and rural populations can help to support the planning and implementation of appropriate measures to improve health in the community. This study aims to determine the factors associated with the health-seeking behavior among Malaysian adults in urban and rural areas who reported sickness. This study used data of Malaysian adults aged 18 years and over from the National Health and Morbidity Survey 2019; a cross-sectional, national household survey that targeted all non-institutionalized residents in Malaysia. Respondent's characteristics and health-seeking behavior were described using complex sample descriptive statistics. Multivariable logistic regression analysis was conducted to examine the association between potential factors (sociodemographic characteristics, enabling, and health need) and health-seeking behaviors (seeking treatment from healthcare practitioners and self-medication). A total of 10,484 respondents, estimated to represent 18.9 million Malaysian adults aged 18 years and over, were included in the analysis. Prevalence of seeking treatment from healthcare practitioners and self-medication among Malaysian adults with self-reported sickness were $57.3 \%$ and $23.3 \%$, respectively. Self-reported sickness among both the urban and rural populations who rated their health as poor to very poor was more likely to seek treatment than those who rated good to excellent. However, among the urban population, those who rated their health as poor to very poor were less likely to self-medicate. Among the urban population, government employees were more likely to seek treatment, and being without formal education significantly increased the likelihood to self-medicate. Among the rural population, those with at least one long-term condition were more likely to seek treatment than those with none. Understanding the factors which influence health-seeking behavior among the urban and rural population could close the gaps in healthcare utilization among the population in Malaysia.

Keywords: health-seeking behavior; urban population; rural population; adult; self-medication; healthcare provider; Malaysia

\section{Introduction}

Health-seeking behavior is one of the major determinants of health outcomes in a community. It determines how health services are used which influences population health outcomes [1]. Health or care-seeking behavior is defined as any action undertaken by individuals who perceive themselves to have a health problem or to be ill to find an appropriate remedy [2]. Individual attributes, the essence of the community in which a person resides, and the relationship between individual and environmental factors are all linked to the health-seeking behavior of an individual [3]. 
There has been a growing interest in research related to health-seeking behaviors over the years locally and internationally [4,5]. Studies conducted locally among the urban population found that $63.5 \%$ of participants used self-medication for minor ailments [6], $85 \%$ consumed over-the-counter (OTC) medications [7], while $67 \%$ chose to consult the physician when they experienced any health problems [8]. A national study in Brazil indicated that the prevalence of use of medicine via self-medication was $18.3 \%$ [9]. Another study among the rural and urban population of Karachi, Pakistan reported that $93 \%$ of the respondents had practiced self-medication [10].

Several theoretical models explain health behaviors; Evans and Stoddart Model [11], Health Belief Model [12], Grossman Model of Health Demand [13], and Andersen's Behavioral Model [14]. Andersen's Behavioral Model of Health Care Utilization is one of the most widely used for predicting health-seeking behaviors due to the convenience of application and popularity in modeling studies involving healthcare accessibility and utilization [14].

Geographic location has a significant influence on the accessibility to healthcare services $[15,16]$, and access to healthcare is reported as one of the many pivotal factors contributing to the gap in health equity among the urban and rural populations [17]. The Malaysian health system is based on a geographically widespread healthcare delivery system designed to provide the entire population with access to public health services, both in the rural and urban localities [18]. An urban area in Malaysia is classified as a gazetted area with a combined population of 10,000 or more, whereas a rural area is defined as a gazetted area with a combined population of less than 10,000 [19]. The equitable healthcare financing and structured public healthcare system in Malaysia [20] does not inherently translate to equitable access because geographical barriers exist [21], among other factors.

In the health sector, access and utilization are interrelated concepts, with access playing a critical role in the utilization of healthcare services. Access to health care was defined as "actual use of personal health services and everything that facilitates or impedes their use" [22]. According to Levesque's Conceptual Framework of Access to Health, the five dimensions of accessibility are approachability, acceptability, availability/accommodation, affordability, and appropriateness [23]. Internationally, research has documented differences in access to and utilization of health care services between urban and rural populations, which consequently affected their health outcomes. Rural patients experienced more barriers to access health care (i.e., distance, travel time, transportation, infrastructure building, medical resources, staff distribution, and clinic distribution) as compared to their urban counterparts [18,24-29], which resulted in having to restart the care-seeking process, inappropriate use of emergency departments, unmet need for care, or health problem exacerbation $[25,30]$.

The introduction of the New Economic Policy in the 1970s has increased the urbanization rate from $26.8 \%$ in 1970 to $71.0 \%$ in 2010 , which were expected to rise to $76.6 \%$ in 2020 and $88.0 \%$ in 2050 [31]. As of 2020, the Malaysian public healthcare system has a distribution of 3171 clinics and 154 hospitals throughout the country, which also provide mobile clinic services to remote areas. There were 7988 registered clinics and a total of 250 licensed hospitals, maternity homes, nursing homes, and hospices in the private healthcare facilities in Malaysia, which are mostly concentrated in the urban areas [18,32,33]. The allocation of healthcare services and resources within the public sector was uneven, favoring urban clinics heavily [24]. Compared to rural areas, urban areas have a greater density of primary care clinics and health workers per capita (2.2 clinics and 15.1 healthcare practitioners per 10,000 population in urban areas versus 1.1 clinics and 11.7 healthcare practitioners per 10,000 population in rural areas) [24].

Malaysia has a dual healthcare system, where the main providers of healthcare are public and private sectors [34]. In ensuring efficiency through decentralization, the hierarchical organization structure of the Ministry of Health $(\mathrm{MOH})$ Malaysia is stratified into the Federal, State, and District levels [35]. Funded through general revenue, the public sector aims to provide universal access with a focus on low-cost but high-benefit health care programs. To keep up with the population growth, especially in urban areas, the dual 
healthcare system has developed with the private sector serving mostly urban regions and better-off patients with fee-for-service primary and secondary care, while the public sector maintains its social equity mission, including primary care services for poor and rural populations [34]. As an expansion of healthcare services in Malaysia, pharmacy practice has also evolved beyond traditional dispensing, from a product-oriented to patientoriented service where in-house pharmacists provided counseling in drug safety, poison information, and medication understanding. Some community pharmacies offered other services such as blood pressure monitoring, chronic disease screening [36], and weight management $[37,38]$. Although the expansion of community pharmacies in Malaysia means people may have more access to over-the-counter medicine, the $\mathrm{MOH}$ has implemented rules about prescription-only medicines, such as antibiotics. Other studies found that the causes of misuse and overuse leading to antibiotic resistance are various [39], and largely due to antibiotics dispensed without a prescription [40]. National guidelines on antibiotics have also been made accessible to the public and healthcare practitioners [41,42].

The duality of Malaysia's healthcare system is further magnified with the practice of both conventional Western medicine (also referred to as modern medicine) as well as traditional and complementary medicine (T\&CM) as part of its healthcare services [18,43,44]. Under the enforcement of the T\&CM Act, T\&CM such as herbal therapy, acupuncture, and traditional massages were also incorporated in some public \& private hospitals as supplementary treatment modalities [43,45]. This was in line with the World Health Organization's efforts to maximize the potentials of safe and quality T\&CM services as a complement to modern medicine among its member states, to achieve holistic healthcare as part of the Universal Health Coverage (UHC) initiative [43,46,47].

Understanding health-seeking behavior and its associated factors would enable health systems to review strategies to accommodate healthcare expectations in the community [48]. Although this knowledge is vital in the proper designing of healthcare policies, very few studies have been conducted at the national level to explore the factors which influence health-seeking behavior among the adult population in the urban and rural areas in Malaysia. In this study, we aim to (1) determine the characteristics of respondents based on locality (urban-rural), (2) determine the prevalence of sick Malaysian adults based on locality, and (3) determine the factors associated with the health-seeking behavior of Malaysian adults who reported sickness, according to locality.

\section{Materials and Methods}

\subsection{Study Design and Participants}

The data for this study was obtained from the National Health and Morbidity Survey (NHMS) 2019, a cross-sectional household survey with a two-stage stratified sampling method to ensure national representativeness. It was conducted among the population in Malaysia who were non-institutionalized and residing in the selected households for at least 2 weeks before the data collection. States and federal territories constituted the primary stratum, and urban and rural areas within the states were considered the secondary stratum. The sampling frame for this survey was provided by the Department of Statistics Malaysia using the National Population and Housing Census 2010. All 13 states and 3 federal territories were included in this survey. Within each state, the required number of Enumeration Blocks (EBs) from urban and rural areas were randomly chosen. First stage sampling involved a random selection of $463 \mathrm{EBs}$ ( 350 urban and 113 rural) from the total EBs in Malaysia (over 75,000 EBs) via a probability proportional to size sampling technique. Subsequently, in each selected EB, 14 Living Quarters (LQs) were selected during the secondstage sampling. All households within the selected LQs and all members in the households were invited to participate in this survey. A total of 5365 LQs were successfully visited giving an LQ response rate of $92.6 \%$ and a total of 16,688 respondents were successfully interviewed giving an individual response rate of $90.0 \%$. The overall response rate for this community-based survey was therefore $83.4 \%$. A detailed methodology and sampling design of the survey is described in the NHMS 2019 official report [44]. 
A total of 10,933 Malaysian adults aged 18 years and over participated in the survey. Only data of respondents with complete responses on potential predictors (sociodemographic characteristics, enabling, and health need factors), experienced acute health problems, and health-seeking behaviors (seeking treatment from a healthcare practitioner and self-medication) were included in this study. In this study, the proportion of missing data was $4.11 \%(n=449)$ and the missing data proportion of less than $5 \%$ was acceptable for complete case analysis [49]. When a preliminary analysis of all respondents was conducted, including those with missing data, no differences in results were observed.

\subsection{Data Collection}

In NHMS 2019, data were collected from July to October 2019 by trained research assistants, via face-to-face interviews using a validated questionnaire [50,51]. The questionnaire was programmed into an application and uploaded onto digital tablets as mobile data collection tools. The tablets were used to collect data, store and back up data in the SD cards, and upload data to the central system. To ensure the minimum sample size required is achieved, vacant or closed houses during the first visit were revisited up to at least three times. The tenets of the Declaration of Helsinki were followed during the study. Written informed consent was obtained from all participants before the interviews. The Medical Research and Ethics Committee (MREC), MOH Malaysia granted permission to carry out the National Health and Morbidity Survey 2019 (NMRR-18-3085-44207).

\subsection{Study Variables}

\subsubsection{Andersen's Behavioral Model of Health Care Utilization}

Andersen's Behavioral Model of Health Care Utilization was adapted into this study for its convenience of application and popularity in modeling studies involving healthcare accessibility and utilization. The model suggests that the health-seeking behavior of individuals is influenced by three groups of factors: sociodemographic characteristics, enabling, and health needs. Sociodemographic characteristics describe the tendency to use the services (i.e., sex, ethnicity, age, education level, and marital status), enabling factors describes the resources available to use the health services and facilities (i.e., wealth status, social support, and access to health resources), and health need factors represent perceived need for healthcare services [14].

\subsubsection{Dependent Variables}

In this study, there are two dependent variables included which are: (1) seeking treatment from healthcare practitioners among those who reported sickness for the last 2 weeks before the interview, and (2) self-medication among those who reported sickness for the last 2 weeks before the interview. Those who reported sickness in the last 2 weeks before the interview were respondents who answered "yes" to the question "In the last 2 weeks, did you experience any of the following health problems such as fever, sore throat, difficulty in swallowing, running nose or blocked nose, cough, and others"

Those who answered "yes", were then asked their health-seeking behavior (yes or no) based on the question "In the last 2 weeks, did you seek treatment/medication or advice from healthcare practitioners?" and "In the last 2 weeks, did you take medicine without advice from healthcare practitioners?" In this study, the term seeks treatment was used to refer to "seek treatment/medication or advice from healthcare practitioners" in short. Healthcare practitioners refer to modern healthcare practitioners including community pharmacists as well as traditional and complementary medicine practitioners (e.g., spiritual healer, Chinese herbalist, Ayurvedic practitioner, and Islamic medicine practitioner). Self-medication was used to refer to "take medicine without advice from healthcare practitioners" 


\subsubsection{Independent Variables}

Sociodemographic Characteristics

In this study, sociodemographic variables included were: sex (male or female); ethnicity (Malay or Non-Malay); age (a continuous variable, grouped into 18-34, 35-59, or $60+$ years); education level (no formal education, primary, secondary, or tertiary education); and marital status (single, married, or widow(er)/divorced/separated). The age of respondents in years was grouped into "18-34", "35-59", and "60+ years" based on age distribution pattern. Education levels were categorized into four groups: no formal education, primary, secondary, and tertiary education. Respondents who had never been to school to get any form of education or did not complete primary school were categorized into 'no formal education, while those who completed Standard Six were categorized as 'primary' education level. 'Secondary' education level represented those with at least five years of schooling at secondary school, whereas 'tertiary' education level represented those who completed Form Six or received certificates, diplomas, or academic degrees.

\section{Enabling Factors}

The enabling factors included were: employment status (government employee, private employee, self-employed, or unemployed); income (quintile 1 (Q1), quintile 2 (Q2), quintile $3(\mathrm{Q} 3)$, quintile $4(\mathrm{Q} 4)$, or quintile $5(\mathrm{Q} 5)$ ), calculated based on total monthly household income and then were grouped into quintiles; and healthcare coverage (yes or no). Q1 represents the poorest $20 \%$ of the population and Q5, the $20 \%$ richest. Healthcare coverage was defined as having supplementary financial coverage for health care such as government employees' health benefits, pensioner cards, government-specific health fund, personal health insurance, employer-sponsored insurance, and panel clinic.

\section{Health Need Factors}

Proxy measures for health needs included were: self-rated health (good to excellent, fair, or poor to very poor); and presence of at least one long-term condition (yes or no), assessed from the questions "Have you ever been told by a doctor or assistant medical officer that you have diabetes?", "Have you ever been told by a doctor or assistant medical officer that you have high blood pressure?" and "Have you ever been told by a doctor or assistant medical officer that you have high cholesterol?" For the analysis, respondents who answered at least one "yes" to either one of the conditions, were coded as "yes" to "presence of at least one long-term condition"

\subsection{Statistical Analysis}

Secondary data analysis was conducted using STATA version 14 (Stata Corp, College Station, TX, USA). Complex sample descriptive statistics were used to illustrate the sociodemographic, enabling, and health need characteristics of the respondents, according to their locality (urban-rural). Sample weights and study design were taken into consideration using a complex sampling design in all data analyses. The products of the inverse of the probability of sampling, a non-response adjustment factor, and a post-stratification adjustment by age, gender, and ethnicity were used to calculate the weight used for estimation.

Comparison of characteristics between urban and rural populations was performed using the chi-square test. Univariate with the chi-square test and multivariable logistic regression analysis, which presented as crude odds ratios (COR) and adjusted odds ratios (AOR) with $95 \%$ confidence intervals (CI), were used to predict characteristics of those who sought treatment from healthcare practitioners, and those who self-medicated, stratified by urban-rural locality. All variables with a $p$-value $<0.25$ in the univariate analysis were considered as predictive variables and entered into multivariable regression analysis [52]. The multivariable analysis was performed for urban and rural separately to examine the predictive factors for seeking treatment and self-medication using four models while adjusting for all other potential covariates such as sociodemographic characteristics, enabling, and health need factors. The AOR with a 95\% confidence interval was determined where 
$p$-value $<0.05$ was considered statistically significant. The goodness of fit model was tested using Hosmer-Lemeshow statistics, and $p$-value $>0.05$ was considered as a good fit.

\section{Results}

A total of 10,484 respondents representing 18.9 million population were included in the analysis. The respondents comprised of urban population (76.1\%) and rural population (23.9\%). Table 1 shows the sociodemographic characteristics, enabling, and health need factors of the respondents, stratified by locality. Both urban and rural populations had significant differences in all factors, except marital status and the presence of at least one long-term condition.

Table 2 presents the prevalence of Malaysian adults who reported sickness. The overall prevalence of Malaysian adults who reported sickness was $16.1 \%$. Of these, more than half $(57.3 \%)$ sought treatment from healthcare practitioners, and about a quarter $(23.3 \%)$ self-medicated. The prevalence of Malaysian adults in the rural areas who reported sickness $(17.6 \%)$ was higher than the urban adults $(15.6 \%)$. There were significant differences in the prevalence of those who reported sickness by different sociodemographic characteristics. Among the urban population, a higher prevalence of self-reported sickness was seen among females. Among the rural population, a higher prevalence of self-reported sickness was seen among non-Malays, aged 60 and over, those without formal education as well as a widow(er)/divorced/separated. Prevalence of self-reported sickness among those who self-rated their health as poor to very poor and those with at least one long-term condition was higher among both the urban and rural populations. Among those who reported sickness, more than half $(57.3 \%)$ sought treatment from healthcare practitioners, and about a quarter $(23.3 \%)$ self-medicated.

Table 3 displays the results of the logistic regression analysis of health-seeking behaviors, with COR and AOR, and their CIs and $p$-values. The model I and II assessed the factors associated with seeking treatment among self-reported sick adults in urban and rural localities, respectively. The multivariable logistic regression revealed that employment status and self-rated health were significantly positively associated with seeking treatment among the urban population. Among urban dwellers, government employees were about 2 times (AOR $=1.82,95 \%$ CI: 1.01-3.27) more likely to seek treatment than those who were self-employed. Urban dwellers who rated their health as poor to very poor were about 3 times (AOR $=2.94,95 \%$ CI: 1.47-5.88) more likely to seek treatment than those who rated good to excellent.

Whereas among the rural population, self-rated health and presence of any longterm conditions were significantly positively associated with seeking treatment. Urban dwellers who rated their health as poor to very poor were about 4 times $(\mathrm{AOR}=3.68$, 95\% CI: 1.36-9.97) more likely to seek treatment than those who rated good to excellent health, whereas those with at least one long-term condition were about 2 times ( $\mathrm{AOR}=2.06$, 95\% CI: 1.23-3.45) more likely to seek treatment than those with none.

Model III and IV assessed the factors associated with self-medication among selfreported sick adults in urban and rural localities, respectively. The regression revealed that education levels were significantly positively associated with self-medication among urban dwellers, where being without formal education significantly increased the likelihood of about 4.3 times ( $\mathrm{AOR}=4.29,95 \% \mathrm{CI}$ : 1.81-10.17) to self-medicate. Whereas self-rated health was significantly negatively associated with self-medication among the urban population. Urban dwellers who rated their health as poor to very poor were less likely ( $\mathrm{AOR}=0.40$, 95\% CI: 0.16-0.98) to self-medicate than those who rated good to excellent. However, in terms of self-medication among those who reported sickness in the rural locality, there was no significant association found. The Hosmer-Lemeshow test showed the goodness-fit of the models $(p>0.05)$. Thus, these models were considered a good fit. 
Table 1. Characteristics of respondents, stratified by urban-rural locality $(\mathrm{N}=10,484)$.

\begin{tabular}{|c|c|c|c|c|c|c|}
\hline \multirow{3}{*}{ Characteristics } & \multirow{3}{*}{$\begin{array}{c}\text { Count, } n \\
\text { (Unweighted) }\end{array}$} & \multirow{3}{*}{$\begin{array}{c}\text { Estimated } \\
\text { Population, } n \\
\text { (Weighted) }\end{array}$} & \multirow{3}{*}{$\begin{array}{l}\% \text { Weighted } \\
\quad(95 \% \text { CI })\end{array}$} & \multicolumn{2}{|c|}{ Locality } & \multirow{2}{*}{$p$-Value } \\
\hline & & & & Urban & Rural & \\
\hline & & & & $\begin{array}{l}\text { \% Weighted } \\
\text { (95\% CI) }\end{array}$ & $\begin{array}{l}\text { \% Weighted } \\
\text { (95\% CI) }\end{array}$ & \\
\hline \multicolumn{7}{|l|}{ Sociodemographic } \\
\hline \multicolumn{7}{|l|}{ Sex } \\
\hline Male & 4905 & $9,116,299$ & $48.2(47.0-49.5)$ & $49.8(48.3-51.3)$ & $43.2(40.9-45.5)$ & $<0.001 *$ \\
\hline Female & 5579 & $9,778,742$ & $51.8(50.5-53.0)$ & $50.2(48.7-51.7)$ & $56.8(54.5-59.1)$ & \\
\hline \multicolumn{7}{|l|}{ Ethnicity } \\
\hline Malay & 7237 & $10,810,187$ & $57.2(52.9-61.4)$ & $52.7(47.5-57.9)$ & $71.5(65.8-76.5)$ & $<0.001$ * \\
\hline Non-Malay & 3247 & $8,084,854$ & $42.8(38.6-47.1)$ & $47.3(42.1-52.5)$ & $28.5(23.5-34.2)$ & \\
\hline \multicolumn{7}{|l|}{ Age (years) } \\
\hline $18-34$ & 3257 & $7,788,423$ & $41.2(39.7-42.7)$ & $41.0(39.2-42.9)$ & $41.8(39.4-44.1)$ & $<0.001 *$ \\
\hline $35-59$ & 4848 & $7,966,079$ & $42.2(40.7-43.6)$ & $43.6(41.9-45.3)$ & $37.6(35.4-39.8)$ & \\
\hline $60+$ & 2379 & $3,140,539$ & $16.6(15.4-18.0)$ & $15.4(13.9-17.0)$ & $20.6(18.4-23.1)$ & \\
\hline \multicolumn{7}{|l|}{ Education level } \\
\hline No formal & 526 & 704,841 & $3.7(3.2-4.3)$ & $2.7(2.2-3.4)$ & $7.0(6.0-8.1)$ & $<0.001 *$ \\
\hline Primary & 2179 & $3,285,633$ & $17.4(16.1-18.7)$ & $14.7(13.3-16.3)$ & $25.8(23.4-28.5)$ & \\
\hline Secondary & 5077 & $9,516,574$ & $50.4(48.7-52.1)$ & $50.7(48.6-52.8)$ & $49.4(46.8-52.0)$ & \\
\hline Tertiary & 2702 & $5,387,993$ & $28.5(26.6-30.5)$ & $31.9(29.5-34.4)$ & $17.8(15.2-20.6)$ & \\
\hline \multicolumn{7}{|l|}{ Marital status } \\
\hline Single & 2213 & $5,349,399$ & $28.3(26.7-30.0)$ & $28.6(26.7-30.7)$ & $27.3(24.7-30.0)$ & 0.147 \\
\hline Married & 7116 & $11,932,813$ & $63.2(61.3-64.9)$ & $63.3(61.0-65.4)$ & $62.8(59.9-65.6)$ & \\
\hline Widow(er)/Divorcee/Separated & 1155 & $1,612,829$ & $8.5(7.7-9.4)$ & $8.1(7.7-9.4)$ & $9.9(8.6-11.4)$ & \\
\hline Enabling & & & & & & \\
\hline \multicolumn{7}{|l|}{ Employment status } \\
\hline Government & 1177 & $1,511,788$ & $8.0(7.1-9.1)$ & $8.2(7.1-9.5)$ & $7.4(5.9-9.2)$ & $<0.001 *$ \\
\hline Private & 2873 & $6,351,279$ & $33.6(31.7-35.6)$ & $38.1(35.8-40.6)$ & $19.3(16.6-22.3)$ & \\
\hline Self-employed & 1977 & $3,493,565$ & $18.5(17.2-19.9)$ & $16.5(15.0-18.1)$ & $24.9(22.4-27.5)$ & \\
\hline Unemployed & 4457 & $7,538,409$ & $39.9(38.3-41.5)$ & $37.2(45.4-51.5)$ & $48.5(45.4-51.5)$ & \\
\hline \multicolumn{7}{|l|}{ Household income quintile } \\
\hline Q1 (20\% poorest $)$ & 2277 & $3,932,420$ & $20.8(19.3-22.4)$ & $17.1(15.5-18.9)$ & $32.6(29.2-36.3)$ & $<0.001$ * \\
\hline Q2 & 1992 & $3,398,139$ & $18.0(16.4-19.7)$ & $15.7(13.9-17.7)$ & $25.3(22.4-28.4)$ & \\
\hline Q3 & 2003 & $3,754,531$ & $19.9(17.9-22.0)$ & $20.4(18.0-23.0)$ & $18.3(15.7-21.3)$ & \\
\hline$\widehat{\mathrm{Q}} 4$ & 2093 & $3,786,071$ & $20.0(18.2-22.0)$ & $22.1(19.9-24.6)$ & $13.3(10.9-16.2)$ & \\
\hline Q5 (20\% richest) & 2119 & $4,023,880$ & $21.3(18.9-23.9)$ & $24.7(21.8-27.9)$ & $10.5(8.0-13.6)$ & \\
\hline \multicolumn{7}{|l|}{$\begin{array}{l}\text { Covered by any healthcare } \\
\text { coverage }\end{array}$} \\
\hline Yes & 5609 & $10,439,085$ & $55.2(53.1-57.4)$ & $60.6(57.9-63.1)$ & $38.4(34.8-42.0)$ & $<0.001^{*}$ \\
\hline No & 4875 & $8,455,956$ & $44.8(42.6-46.9)$ & $39.4(36.9-42.1)$ & $61.6(58.0-65.2)$ & \\
\hline \multicolumn{7}{|l|}{ Health need } \\
\hline \multicolumn{7}{|l|}{ Self-rated health } \\
\hline Excellent \& Good & 7856 & $14,814,257$ & $78.4(76.9-79.9)$ & $80.0(78.3-81.7)$ & $73.2(70.1-76.2)$ & $<0.001$ * \\
\hline Fair & 2371 & $3,689,380$ & $19.5(18.2-21.0)$ & $17.9(16.4-19.6)$ & $24.7(22.0-27.5)$ & \\
\hline Poor \& Very Poor & 257 & 391,404 & $2.1(1.7-2.5)$ & $2.1(1.7-2.6)$ & $2.1(1.5-2.8)$ & \\
\hline \multirow{2}{*}{\multicolumn{7}{|c|}{$\begin{array}{l}\text { Presence of at least one } \\
\text { long-term condition }\end{array}$}} \\
\hline & & & & & & \\
\hline Yes & 3148 & $4,639,737$ & $24.6(23.3-25.8)$ & $24.2(22.7-25.7)$ & $25.7(24.0-27.5)$ & 0.211 \\
\hline No & 7336 & $14,255,304$ & $75.4(74.2-76.7)$ & $75.8(74.3-77.3)$ & $74.3(72.5-76.0)$ & \\
\hline
\end{tabular}

$n$, count; \%, percentage; CI, Confidence Interval; Q, Quintile. $p$-values were obtained by chi-square test; * indicates a statistical significance. Healthcare coverage refers to government employees' health benefits, pensioner cards, government-specific health funds, personal health insurance, employer-sponsored insurance, and panel clinic. Long-term condition refers to presence of any non-communicable diseases (diabetes, hypertension or hypercholesterolemia). 
Table 2. Prevalence of those who reported sickness among Malaysian adults, stratified by urbanrural locality.

\begin{tabular}{|c|c|c|c|c|c|c|c|c|}
\hline \multirow[b]{2}{*}{ Characteristics } & \multicolumn{2}{|c|}{ Overall $(\mathrm{N}=10,484)$} & \multicolumn{3}{|c|}{ Urban $(n=6288)$} & \multicolumn{3}{|c|}{ Rural $(n=4196)$} \\
\hline & Count & $\begin{array}{l}\text { \% Weighted } \\
\text { (95\% CI) }\end{array}$ & Count & $\begin{array}{l}\text { \% Weighted } \\
\text { (95\% CI) }\end{array}$ & $p$-Value & Count & $\begin{array}{l}\% \text { Weighted } \\
(95 \% \text { CI })\end{array}$ & $p$-Value \\
\hline OVERALL & 1946 & $16.1(14.8-17.4)$ & 1187 & $15.6(14.1-17.3)$ & - & 759 & $17.6(15.5-19.9)$ & - \\
\hline \multicolumn{9}{|l|}{ Sociodemographic } \\
\hline Male & 778 & $14.1(12.6-15.7)$ & 460 & $13.3(11.7-15.2)$ & \multirow[t]{2}{*}{$<0.001^{*}$} & 318 & 16.7 (14.1-19.7) & \multirow[t]{2}{*}{0.441} \\
\hline Female & 1168 & $17.9(16.2-19.8)$ & 727 & $17.8(15.8-20.1)$ & & 441 & $18.2(15.5-21.3)$ & \\
\hline \multicolumn{9}{|l|}{ Ethnicity } \\
\hline Malay & 1349 & $15.8(14.3-17.5)$ & 793 & $16.4(14.5-18.5)$ & \multirow[t]{2}{*}{0.283} & 556 & $14.5(12.2-17.1)$ & \multirow[t]{2}{*}{$<0.001$ * } \\
\hline Non-Malay & 597 & $16.4(14.3-18.8)$ & 556 & 14.7 (12.4-17.3) & & 203 & $25.3(21.0-30.3)$ & \\
\hline \multicolumn{9}{|l|}{ Age (years) } \\
\hline $18-34$ & 580 & $15.3(13.5-17.3)$ & 391 & $15.6(13.4-18.1)$ & \multirow[t]{3}{*}{0.407} & 189 & $14.3(11.4-17.8)$ & \multirow[t]{3}{*}{$0.005 *$} \\
\hline $35-59$ & 875 & $15.8(14.2-17.6)$ & 559 & $15.0(13.1-17.0)$ & & 316 & $18.8(15.8-22.2)$ & \\
\hline $60+$ & 491 & $18.7(16.4-21.4)$ & 237 & $17.4(14.4-20.9)$ & & 254 & $21.9(18.5-25.8)$ & \\
\hline \multicolumn{9}{|l|}{ Education level } \\
\hline No formal & 120 & $23.0(18.1-28.8)$ & 40 & $17.0(10.8-25.8)$ & \multirow[t]{4}{*}{0.148} & 80 & $30.4(23.3-38.7)$ & \multirow[t]{4}{*}{$<0.001$ * } \\
\hline Primary & 438 & $19.3(17.0-21.9)$ & 209 & $18.4(15.3-21.9)$ & & 229 & $20.9(17.8-24.5)$ & \\
\hline Secondary & 871 & $14.6(13.1-16.2)$ & 527 & $14.4(12.6-16.4)$ & & 344 & $15.4(12.9-18.2)$ & \\
\hline Tertiary & 517 & $15.8(13.6-18.3)$ & 411 & $16.2(13.7-19.1)$ & & 106 & $13.7(10.4-18.0)$ & \\
\hline \multicolumn{9}{|l|}{ Marital status } \\
\hline Single & 358 & $13.6(11.5-16.0)$ & 246 & $13.8(11.3-16.7)$ & \multirow[t]{3}{*}{0.060} & 112 & $12.9(9.3-17.6)$ & \multirow[t]{2}{*}{0.010 * } \\
\hline Married & 1326 & $16.6(15.1-18.1)$ & 794 & $15.9(14.3-17.7)$ & & 532 & $18.6(16.0-12.5)$ & \\
\hline $\begin{array}{l}\text { Widow(er)/Divorcee/Separated } \\
\text { Enabling }\end{array}$ & 262 & $20.7(17.3-24.7)$ & 147 & $19.5(15.3-24.5)$ & & 115 & $23.9(18.8-29.9)$ & \\
\hline \multicolumn{9}{|l|}{ Employment status } \\
\hline Government & 268 & $17.9(14.6-21.8)$ & 204 & $17.5(13.6-22.1)$ & 0.745 & 64 & $19.5(14.2-26.2)$ & 0.558 \\
\hline Private & 480 & $15.0(12.8-17.4)$ & 334 & $14.9(12.6-17.6)$ & & 146 & $15.3(11.0-20.8)$ & \\
\hline Self-employed & 335 & $16.3(14.1-18.9)$ & 165 & $15.9(13.1-19.3)$ & & 170 & $17.2(14.0-20.9)$ & \\
\hline Unemployed & 863 & $16.5(14.8-18.3)$ & 484 & $15.8(13.7-18.1)$ & & 379 & $18.4(15.7-21.4)$ & \\
\hline $\begin{array}{l}\text { Household income } \\
\text { quintile }\end{array}$ & & & & & & & & \\
\hline Q1 (20\% poorest) & 447 & $17.3(15.2-19.6)$ & 204 & $14.7(12.1-17.9)$ & 0.433 & 243 & $21.5(18.4-25.1)$ & 0.060 \\
\hline Q2 & 362 & $17.1(14.7-19.8)$ & 195 & $17.0(14.0-20.6)$ & & 167 & $17.2(13.7-21.4)$ & \\
\hline Q3 & 378 & $16.4(14.1-19.0)$ & 218 & $16.4(13.6-19.6)$ & & 160 & $16.5(12.7-21.0)$ & \\
\hline$\widehat{\mathrm{Q}} 4$ & 363 & $15.7(13.1-18.6)$ & 254 & $16.5(13.6-19.9)$ & & 109 & $11.5(8.4-15.5)$ & \\
\hline Q5 (20\% richest) & 396 & $14.1(11.8-16.7)$ & 316 & $13.9(11.5-16.6)$ & & 80 & $15.9(8.8-26.9)$ & \\
\hline $\begin{array}{c}\text { Covered by any healthcare } \\
\text { coverage }\end{array}$ & & & & & & & & \\
\hline Yes & 1084 & $16.4(14.7-18.3)$ & 759 & $16.0(14.1-18.1)$ & 0.463 & 325 & $18.7(15.2-22.9)$ & 0.348 \\
\hline No & 862 & $15.6(14.2-17.2)$ & 428 & $15.0(13.1-17.2)$ & & 434 & $16.9(14.9-19.1)$ & \\
\hline Health need & & & & & & & & \\
\hline Self-rated health & & & & & & & & \\
\hline Excellent \& Good & 1099 & $12.3(11.0-13.6)$ & 685 & $12.0(10.6-13.6)$ & $<0.001 *$ & 414 & $13.2(11.0-15.7)$ & $<0.001 *$ \\
\hline Fair & 733 & $28.0(25.4-30.8)$ & 430 & $27.8(24.4-31.5)$ & & 303 & $28.5(24.7-32.7)$ & \\
\hline Poor \& Very Poor & 114 & $47.5(38.8-56.4)$ & 72 & $49.3(38.8-59.9)$ & & 42 & $41.9(28.4-56.7)$ & \\
\hline $\begin{array}{l}\text { Presence of at least one } \\
\text { long-term condition }\end{array}$ & & & & & & & & \\
\hline Yes & 728 & $21.3(19.0-23.7)$ & 417 & $20.4(17.7-23.5)$ & $<0.001 *$ & 311 & 23.7 (20.1-27.7) & $<0.001 *$ \\
\hline No & 1218 & $14.4(13.0-15.9)$ & 770 & $14.1(12.5-15.8)$ & & 448 & $15.5(13.2-18.1)$ & \\
\hline $\begin{array}{l}\text { Health-seeking behavior } \\
\text { Sought treatment from } \\
\text { healthcare practitioner }\end{array}$ & & & & & & & & \\
\hline Yes & 1122 & $57.3(53.7-60.8)$ & 681 & $58.9(24.3-63.3)$ & - & 441 & $52.6(47.6-57.6)$ & - \\
\hline No & 824 & $42.7(39.2-46.3)$ & 506 & $41.1(36.7-45.7)$ & & 318 & $47.4(42.4-52.4)$ & \\
\hline Self-medicated & & & & & & & & \\
\hline Yes & 438 & $23.3(20.2-26.8)$ & 258 & $23.2(19.3-27.7)$ & - & 180 & $23.6(19.3-28.4)$ & - \\
\hline No & 1508 & 76.7 (73.2-79.8) & 929 & 76.8 (72.3-80.7) & & 579 & $76.4(71.6-80.7)$ & \\
\hline
\end{tabular}

$n$, count; \%, percentage; CI, Confidence Interval; Q, Quintile. p-values were obtained by chi-square test; * indicates a statistical significance. Healthcare coverage refers to government employees' health benefits, pensioner card, government-specific health fund, personal health insurance, employer-sponsored insurance, and panel clinic. Long-term condition refers to presence of any non-communicable diseases (diabetes, hypertension or hypercholesterolemia). 
Table 3. Logistic regression model for health-seeking behaviors among Malaysian adults who reported sickness, stratified by urban-rural locality.

\begin{tabular}{|c|c|c|c|c|c|c|c|c|}
\hline \multirow{3}{*}{ Factors } & \multicolumn{4}{|c|}{ Sought Treatment from Healthcare Practitioner } & \multicolumn{4}{|c|}{ Self-Medicated } \\
\hline & \multicolumn{2}{|c|}{ Model I-Urban } & \multicolumn{2}{|c|}{ Model II-Rural } & \multicolumn{2}{|c|}{ Model III-Urban } & \multicolumn{2}{|c|}{ Model IV-Rural } \\
\hline & $\begin{array}{l}\text { Crude OR } \\
(95 \% \text { CI })\end{array}$ & $\begin{array}{l}\text { Adjusted OR } \\
(95 \% \mathrm{CI})\end{array}$ & $\begin{array}{l}\text { Crude OR } \\
(95 \% \mathrm{CI})\end{array}$ & $\begin{array}{l}\text { Adjusted OR } \\
(95 \% \mathrm{CI})\end{array}$ & $\begin{array}{l}\text { Crude OR } \\
(95 \% \text { CI })\end{array}$ & $\begin{array}{l}\text { Adjusted OR } \\
\quad(95 \% \mathrm{CI})\end{array}$ & $\begin{array}{l}\text { Crude OR } \\
(95 \% \text { CI })\end{array}$ & $\begin{array}{l}\text { Adjusted OR } \\
(95 \% \mathrm{CI})\end{array}$ \\
\hline \multicolumn{9}{|l|}{ Sex } \\
\hline Male & 1.00 (ref) & 1.00 (ref) & 1.00 (ref) & & 1.00 (ref) & & 1.00 (ref) & \\
\hline Female & 1.35 & 1.33 & 0.98 & & 1.10 & & 0.98 & \\
\hline \multicolumn{9}{|l|}{ Ethnicity } \\
\hline Malay & 1.00 (ref) & & 1.00 (ref) & & 1.00 (ref) & & 1.00 (ref) & 1.00 (ref) \\
\hline Non-Malay & $\begin{array}{c}0.81 \\
(0.54-1.20)\end{array}$ & & $\begin{array}{c}0.80 \\
(0.52-1.25)\end{array}$ & & $\begin{array}{c}1.20 \\
(0.76-1.88)\end{array}$ & & $\begin{array}{c}0.67 \\
(0.39-1.14)\end{array}$ & $\begin{array}{c}0.66 \\
(0.38-1.13)\end{array}$ \\
\hline \multicolumn{9}{|l|}{ Age (years) } \\
\hline $18-34$ & 1.00 (ref) & & 1.00 (ref) & 1.00 (ref) & 1.00 (ref) & & 1.00 (ref) & 1.00 (ref) \\
\hline $35-59$ & $\begin{array}{c}1.26 \\
(0.90-1.77)\end{array}$ & & $\begin{array}{c}0.90 \\
(0.54-1.52)\end{array}$ & $\begin{array}{c}0.71 \\
(0.41-1.22)\end{array}$ & $\begin{array}{c}0.90 \\
(0.56-1.44)\end{array}$ & & $\begin{array}{c}1.57 \\
(0.89-2.77)\end{array}$ & $\begin{array}{c}1.58 \\
(0.90-2.79)\end{array}$ \\
\hline $60+$ & $\begin{array}{c}1.20 \\
(0.72-1.97)\end{array}$ & & $\begin{array}{c}1.57 \\
(0.82-3.00)\end{array}$ & $\begin{array}{c}0.88 \\
(0.42-1.87)\end{array}$ & $\begin{array}{c}0.93 \\
(0.52-1.65)\end{array}$ & & $\begin{array}{c}0.94 \\
(0.52-1.69)\end{array}$ & $\begin{array}{c}0.93 \\
(0.52-1.66)\end{array}$ \\
\hline \multicolumn{9}{|l|}{ Education level } \\
\hline No formal & $\begin{array}{c}1.53 \\
(0.66-3.55)\end{array}$ & & $\begin{array}{c}0.98 \\
(0.45-2.14)\end{array}$ & & $\begin{array}{c}3.69 \\
(1.51-9.03)^{* *}\end{array}$ & $\begin{array}{l}4.29(1.81- \\
10.17)^{* *}\end{array}$ & $\begin{array}{c}0.73 \\
(0.34-1.57)\end{array}$ & \\
\hline Primary & $\begin{array}{c}1.19 \\
(0.81-1.75)\end{array}$ & & $\begin{array}{c}1.24 \\
(0.71-2.15)\end{array}$ & & $\begin{array}{c}1.46 \\
(0.87-2.46)\end{array}$ & $\begin{array}{c}1.58 \\
(0.93-2.66)\end{array}$ & $\begin{array}{c}0.94 \\
(0.50-1.79)\end{array}$ & \\
\hline Secondary & 1.00 (ref) & & 1.00 (ref) & & 1.00 (ref) & 1.00 (ref) & 1.00 (ref) & \\
\hline Tertiary & $\begin{array}{c}1.29 \\
(0.84-1.98)\end{array}$ & & $\begin{array}{c}1.18 \\
(0.58-2.43)\end{array}$ & & $\begin{array}{c}1.29 \\
(0.78-2.15)\end{array}$ & $\begin{array}{c}1.26 \\
(0.76-2.09)\end{array}$ & $\begin{array}{c}0.64 \\
(0.28-1.47)\end{array}$ & \\
\hline \multicolumn{9}{|l|}{ Marital status } \\
\hline & 1.03 & & 1.02 & & 1.16 & & 1.42 & \\
\hline Single & $(0.68-1.56)$ & & $(0.52-2.00)$ & & $(0.71-1.89)$ & & $(0.77-2.60)$ & \\
\hline Married & 1.00 (ref) & & 1.00 (ref) & & 1.00 (ref) & & 1.00 (ref) & \\
\hline Widow(er)/Divorcee/Separated & $\begin{array}{c}1.01 \\
(0.54-1.92)\end{array}$ & & $\begin{array}{c}1.33 \\
(0.64-2.73)\end{array}$ & & $\begin{array}{c}1.03 \\
(0.58-1.84)\end{array}$ & & $\begin{array}{c}1.19 \\
(0.56-2.52)\end{array}$ & \\
\hline \multicolumn{9}{|l|}{ Employment status } \\
\hline Government & $\begin{array}{c}1.92 \\
(1.07-3.43)\end{array}$ & $\begin{array}{c}1.82 \\
(1.01-3.27) *\end{array}$ & $\begin{array}{c}0.83 \\
(0.40-1.71)\end{array}$ & & $\begin{array}{c}0.98 \\
(0.45-2.12)\end{array}$ & & $\begin{array}{c}0.59 \\
(0.20-1.72)\end{array}$ & \\
\hline Private & $\begin{array}{c}1.29 \\
(0.80-2.09)\end{array}$ & $\begin{array}{c}1.34 \\
(0.84-2.16)\end{array}$ & $\begin{array}{c}0.92 \\
(0.45-1.91)\end{array}$ & & $\begin{array}{c}1.13 \\
(0.63-2.05)\end{array}$ & & $\begin{array}{c}0.50 \\
(0.23-1.11)\end{array}$ & \\
\hline Self-employed & 1.00 (ref) & 1.00 (ref) & 1.00 (ref) & & 1.00 (ref) & & 1.00 (ref) & \\
\hline Unemployed & $\begin{array}{c}1.59 \\
(1.05-2.41)\end{array}$ & $\begin{array}{c}1.31 \\
(0.83-2.05)\end{array}$ & $\begin{array}{c}1.24 \\
(0.72-2.14)\end{array}$ & & $\begin{array}{c}1.02 \\
(0.60-1.74)\end{array}$ & & $\begin{array}{c}0.66 \\
(0.32-1.37)\end{array}$ & \\
\hline \multicolumn{9}{|l|}{ Household income quintile } \\
\hline Q1 (20\% poorest) & $\begin{array}{c}1.01 \\
(0.58-177)\end{array}$ & & $\begin{array}{c}1.88 \\
(0.78-4.56)\end{array}$ & & $\begin{array}{c}1.03 \\
(0.55-1.94)\end{array}$ & & $\begin{array}{c}1.43 \\
(0.35-5.78)\end{array}$ & \\
\hline & $\begin{array}{c}0.30-1.71) \\
0.76\end{array}$ & & $\begin{array}{l}(0.70-4.00) \\
1.70\end{array}$ & & $\begin{array}{c}(0.55-1.94) \\
1.24\end{array}$ & & $\begin{array}{c}(0.35-5 . / 8) \\
1.45\end{array}$ & \\
\hline Q2 & $(0.46-1.27)$ & & $(0.58-5.03)$ & & $(0.68-2.26)$ & & $(0.37-5.58)$ & \\
\hline Q3 & $\begin{array}{c}0.77 \\
(0.44-1.33)\end{array}$ & & $\begin{array}{c}1.50 \\
(0.61-3.70)\end{array}$ & & $\begin{array}{c}1.27 \\
(0.65-2.47)\end{array}$ & & $\begin{array}{c}1.93 \\
(0.50-7.47)\end{array}$ & \\
\hline & 0.60 & & 1.96 & & 1.34 & & 1.74 & \\
\hline Q4 & $(0.33-1.06)$ & & $(0.63-6.05)$ & & $(0.69-2.60)$ & & $(0.41-7.32)$ & \\
\hline \multirow{2}{*}{\multicolumn{9}{|c|}{$\begin{array}{l}\text { Covered by any healthcare } \\
\text { coverage }\end{array}$}} \\
\hline & & & & & & & & \\
\hline & 0.99 & & 0.69 & 0.77 & 1.13 & & 0.87 & \\
\hline Yes & $(0.70-1.40)$ & & $(0.44-1.07)$ & $(0.47-1.27)$ & $(0.75-1.72)$ & & $(0.49-1.54)$ & \\
\hline No & 1.00 (ref) & & 1.00 (ref) & 1.00 (ref) & 1.00 (ref) & & 1.00 (ref) & \\
\hline \multicolumn{9}{|l|}{ Self-rated health } \\
\hline Excellent \& Good & 1.00 (ref) & 1.00 (ref) & 1.00 (ref) & 1.00 (ref) & 1.00 (ref) & 1.00 (ref) & 1.00 (ref) & \\
\hline Fair & 1.51 & 1.41 & 1.10 & 0.88 & 0.97 & 0.89 & 0.96 & \\
\hline & $(1.09-2.09) *$ & (0.98-2.04) & (0.69-1.75) & (0.54-1.43) & $(0.66-1.42)$ & $(0.62-1.29)$ & (0.55-1.69) & \\
\hline Poor \& Very Poor & $\begin{array}{c}3.04 \\
(1.56-5.90) * *\end{array}$ & $\begin{array}{c}2.94 \\
(1.47-5.88) * * *\end{array}$ & $\begin{array}{c}4.69 \\
(1.69-13.06)^{* *}\end{array}$ & $\begin{array}{c}3.68 \\
(1.36-9.97)^{*}\end{array}$ & $\begin{array}{c}0.49 \\
(0.21-1.13)\end{array}$ & $\begin{array}{c}0.40 \\
(0.16-0.98)\end{array}$ & $\begin{array}{c}0.89 \\
(0.31-2.50)\end{array}$ & \\
\hline \multicolumn{9}{|l|}{$\begin{array}{l}\text { Presence of at least one } \\
\text { long-term condition }\end{array}$} \\
\hline 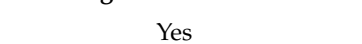 & 1.28 & 1.27 & 2.01 & 2.06 & 0.90 & & 0.82 & \\
\hline & $(0.79-2.07)$ & $(0.81-2.01)$ & $(1.31-3.10)^{* *}$ & $(1.23-3.45)^{* *}$ & $(0.60-1.36)$ & & $(0.49-1.36)$ & \\
\hline No & 1.00 (ref) & 1.00 (ref) & 1.00 (ref) & 1.00 (ref) & 1.00 (ref) & & 1.00 (ref) & \\
\hline
\end{tabular}

Model I assessed the factors associated with seeking treatment among self-reported sick adults in urban locality; Model II assessed the factors associated with seeking treatment among self-reported sick adults in rural locality; Model III assessed the factors associated with self-medication among self-reported sick adults in urban locality Model IV assessed the factors associated with self-medication among self-reported sick adults in rural locality. COR, Crude Odd Ratios; AOR, Adjusted Odd Ratios; CI, Confidence Interval; ref, reference category; Q, Quintile; $*<<0.05 ;$ $* * p<0.01 ;{ }^{* * *} p<0.001$. Goodness of fit for model: Hosmer-Lemeshow statistic: Model I = 0.317; Model II = 0.801; Model III = 1.00; Model IV = 1.00. Healthcare coverage refers to government employees' health benefits, pensioner card, government-specific health fund, personal health insurance, employer-sponsored insurance, and panel clinic. 


\section{Discussion}

This study aimed to determine the characteristics of respondents and prevalence of Malaysian adults who reported sickness based on their urban-rural locality, as well as the factors associated with their health-seeking behaviors. All variables, excluding marital status and the presence of at least one non-communicable disease, were substantially different between the urban and rural populations. The overall prevalence of Malaysian adults who reported sickness was $16.1 \%$, and higher among the rural population as compared to the urban population. Higher prevalence of self-reported sickness among those who self-rated their health as poor to very poor and those with at least one long-term condition were seen among both the urban and rural populations. More than half of those who reported sickness sought treatment from healthcare practitioners, while only about a quarter self-medicated. Self-rated health was one of the factors associated with health-seeking behavior among Malaysian adults who reported sickness from the urban and rural areas.

Overall, less than a fifth of Malaysian adults reported sickness, with the rural populations exhibiting a higher prevalence than those from the urban areas. Similarly, other published studies found that illnesses were more prevalent among the rural population $[27,28,53]$. As an upper-middle-income country, Malaysia's population has benefited from a well-developed health care system, together with improved access to clean water, sanitation, and better child nutrition, which was reinforced through programmes targeted at reducing poverty, increasing literacy, and providing modern infrastructure [54], and these developments may have an effect on the overall population health. Compared to Denmark (about 9 out of 10 respondents reported having experienced at least one symptom) [55] and Hong Kong (46.5\% of the respondents aged between 16 and 54 years reported having any symptoms) [56], Malaysia's population had a better health status in terms of overall prevalence of reported recent illnesses. However, owing to variations in methodology and variables evaluated, these results are not directly comparable.

More than half of those who reported sickness (58.9\% of urban and $52.6 \%$ of rural) sought treatment from healthcare practitioners in the current study and the prevalence was lower among the rural population. Seeking treatment from healthcare practitioners was the first choice of health-seeking behavior reported by previously published studies [29,57]. However, given our results suggest that only slightly more than half of the population sought medical attention, this raises concerns about the proportion of people who did not seek appropriate treatment or care. A study conducted locally reported that $4.9 \%$ and $5.4 \%$ of urban and rural participants, respectively, did not seek treatment when they were sick [29]. Low perception of illness as a major health problem [44,58], low perceived need to seek care [59], work commitment [44], financial constraint $[3,44,59,60]$, and geographical locale [61] were barriers reported in previous studies. As health needs and challenges have changed over the past decade, policymakers must consider the factors that influence people's health-seeking behavior. For the sustainable and equitable provision of health care to the disadvantaged and underserved groups, removing barriers and integrating public and private health services are crucial [62].

Malaysia is among the countries that have achieved UHC, with the vast majority of the population receiving comprehensive public healthcare services [63]. Malaysia, like most other countries, has a two-tiered healthcare system, with a highly subsidized public sector and a fee-for-service private sector [64]. However, this study findings showed that sick rural adults were less likely than their urban counterparts to seek healthcare from a healthcare practitioner. While most studies from other countries have identified sociocultural norms as determinants, distance and proximity to a healthcare facility also was identified as a significant factor for this behavior [65-68]. Within the public sector, the distribution of healthcare facilities and resources heavily favored urban areas [20,25-29,31]. Furthermore, the current study found that a larger percentage of people in rural areas fall into the lower income quintiles. Inadequate access to health care and a lack of income are two reported factors that contribute to the rural population's poor health [65-68]. As the majority of Malaysians with low socioeconomic status came from rural areas [69], this calls 
for more efforts to promote healthcare utilization and enhance accessibility in the remote and rural areas.

This study found that less than a fifth of the population who reported sickness practiced self-medication, which was lower than previous population-based studies $[27,28]$ as well as other local studies [70-72], but higher in study conducted in Sri Lanka (Urban: $12.2 \%$, Rural: $7.9 \%$ ) [73]. This could be because self-medication in Malaysia is more costly compared to seeking treatment from healthcare practitioners, as patients are only charged minimal fee of Malaysian Ringgit (MYR1) [US dollars (USD0.24)] for visits to the public health clinics [74]. Although self-medication assists in the reduction of the burden on medical care, it is linked to many possible risks [70,75-77]. This issue highlights the importance of healthcare practitioners in promoting rational use of medicines, including information on potential side effects, ensuring informed and responsible self-medication [77]. Moreover, public health awareness programmes can be organized as part of larger public health efforts, to help people understand disease processes and positive health behaviors.

According to the World Health Organization, education is one of the key social determinants of health, and addressing it appropriately is essential to promote health and reduce long-standing health inequities [78]. Among the urban population in our study, those with no formal education were more likely to self-medicate than those with higher education levels. The influence of education level on self-medication practice is consistent with a study in Saudi Arabia [79]. In Malaysia, community pharmacies that are strategically and conveniently located in shopping malls and supermarkets [33] led to better access to OTC medications, especially among the urban population where amenities and infrastructure are more readily available. While OTC medications have been shown to be safe and appropriate for use without the supervision of a health care provider, unwanted effects may result if used irresponsibly [80]. Inadequate health literacy among the less educated coupled with easy access to medication may result in serious consequences, which prompts the need to improve health literacy, particularly the negative consequences of self-medication to one's wellbeing. Furthermore, people with a lower degree of education usually have lower health literacy [81]. Thus, the combined effect of easy access to the medications and the higher likelihood of self-medication among those with the lowest educational attainment of the urban population posed a worrying situation. Campaigns such as 'Know Your Medicines', in line with Malaysia's national health agenda, 'Agenda Nasional Malaysia Sihat' (ANMS), advocate the importance of knowing your medications to improve public awareness and empower personal health [82].

Results from our study indicated that self-rated health to be one of the important associated factors in health-seeking behavior. Those who self-rated their health as poor to very poor were more likely to seek care than those who self-rated their health as good to excellent, regardless of locality. Conversely, those who self-rated poor to very poor health was also less likely to self-medicate than those who self-rated good to excellent health among urban population. Published literatures highlighted the association of healthenhancing behaviors, utilization of health services [83,84], and self-medication [79], among those who rated their health as poor, however, the reported results are mixed. Previous studies have established that a single-item measure of self-rated health provides a holistic view of the population's physical and emotional well-being, as well as the ability to predict health-seeking behavior and healthcare use $[50,83,85]$. In our study, those who self-rated their health as poor to very poor were significantly associated with the presence of longterm condition(s) (Table S1), which is consistent with another large-scale study conducted in China [86]. The relationship between presence of long-term condition(s) and poorer health could explain the influence of self-rated health on health-seeking behaviors as the nature of the long-term condition itself, which demands follow-up appointments and a formal prescription to obtain the medications, that may cause this group of people used the health care services and less likely to self-medicate.

Among the urban population, government employees were more likely to seek treatment than those who were self-employed when they were sick. The association between 
occupational status and treatment seeking behavior in this study result is consistent with another study conducted in China, which found that self-employed people were less likely to take remedial action and seek medical help after being ill [87]. This could be attributed to the time constraints to seek treatment when they are sick and financial issues, as selfemployed individuals were more likely to have irregular working hours. Furthermore, their incomes are directly dependent on their work [88]. Additionally, government employees are entitled to a higher number of days for paid sick leave [89] compared to those working in other than the public sector [90].

Rural population with at least one long-term condition were more likely to seek medical treatment than those without, which concurred with previous research that found an association between the presence of chronic illnesses and seeking healthcare services [50,91]. Two-thirds of public primary care clinics in Malaysia are in rural areas [24] and a national cross-sectional study of randomly selected clinics found that doctors in public clinics saw more chronic diseases like hypertension and diabetes, as well as follow-up cases, whereas doctors in private clinics saw more acute and minor illnesses [92]. This occurrence may largely be contributed by the heavily subsidized public healthcare services by the Malaysian government, that also covers the cost of lifelong medications, which is more economical for patients with chronic disease as the nominal fee granted access to the entire spectrum of public healthcare services in the clinics $[18,21,64,74,92]$. This economic factor may have driven private clinics away from the rural areas $[18,24]$. Perceived severity or fear of the consequences of the disease [93] might also be the reason for seeking treatment among the rural population.

This study discovered that gender is not associated with health-seeking behavior among Malaysian adults who reported sickness. Although women were perceived more likely to seek treatment and utilize health services as compared to men [5], previous local studies found that, in general, there was no difference in terms of healthcare utilization across gender [94-96]. In addition, previous national health survey reported that there was no difference in the autonomy of decision making for healthcare between gender [28].

The sample size for this study was large, consisting of 10,484 adults who covered both the urban and rural areas. The proportion of respondents from the urban and rural areas in this survey was very close to Malaysia's real population in the same year [97]. Despite its strengths, this research has a number of limitations. Because of the cross-sectional nature of this research, no causal association between health-seeking behavior and associated factors could be established. Seasonal change could not be measured as the data was collected at just one point in time. Finally, since this analysis used self-reported data on previous events, there is a possibility of recall bias.

\section{Conclusions}

This cross-sectional study showed that sociodemographic, enabling, and health need characteristics were associated with health-seeking behaviors among Malaysian adults who reported sickness from both urban and rural localities, with education level, employment status, self-rated health, and presence of at least one long-term condition as the associated factors. This study revealed gaps in healthcare services and more rooms for improvements despite Malaysia has already achieved UHC status. Understanding the factors which influence health-seeking behavior among the urban and rural population could close the gaps in healthcare utilization among the Malaysian population. Future policies should move towards specific targeted approaches that focus on the rural and vulnerable population, especially regarding access to healthcare services as well as their knowledge and literacy on seeking proper medical care.

Taking care of health should be a culture, a way of life. It should be embedded and be a shared responsibility across all sectors, in line with the Sustainable Development Goals. Social services actors and organizations, which administratively are not under the purview of the MOH Malaysia, are closer to the people's hearts as compared to governmental organizations. Political players are the main drivers with powers to influence the masses. 
Mainstream and social media are also key players in educating the nation regarding health matters. We recommend active two-way engagements, dialogues, and close collaborative efforts with these parties for a shared vision of a healthy nation. We also recommend further in-depth studies to be conducted on factors such as perceived quality of services received, which may provide a deeper understanding on the health-seeking behavior of Malaysia population.

Supplementary Materials: The following are available online at https: / www.mdpi.com/article/ 10.3390 /ijerph19063193/s1, Table S1: Logistic regression model for self-rated health as poor to very poor among Malaysian adults who reported sickness.

Author Contributions: Conceptualization, S.N.M.N., S.J., Y.R.T., I.A.R. and E.H.T.; Data curation, S.N.M.N. and S.J.; Formal analysis, S.N.M.N., S.J. and E.H.T.; Funding acquisition, S.J.; Investigation, S.N.M.N., S.J., Y.R.T. and I.A.R.; Methodology, S.N.M.N., S.J. and E.H.T.; Supervision, E.H.T.; Visualization, S.N.M.N., S.J., Y.R.T., I.A.R. and E.H.T.; Writing-original draft, S.N.M.N., S.J., Y.R.T., I.A.R. and E.H.T.; Writing-review \& editing, S.N.M.N., S.J., Y.R.T., I.A.R. and E.H.T. All authors have read and agreed to the published version of the manuscript.

Funding: This project was registered under National Medical Research Registry (NMRR-18-308544207) and received funding from the National Institutes of Health, Ministry of Health Malaysia research grant. The funding sources had no involvement in study design, study conduct, analyses of the results, writing of this manuscript, and the decision to submit this topic for publication.

Institutional Review Board Statement: The study was conducted in accordance with the Declaration of Helsinki, and approved by the Medical Research and Ethics Committee (MREC), Ministry of Health Malaysia [KKM/NIHSEC/P18-2325(11)] dated 20 December 2018, and was registered in the National Medical Research Register, Ministry of Health Malaysia (NMRR-18-3085-44207).

Informed Consent Statement: Written and informed consent was obtained from each respondent prior to the interviews, and confidentiality of respondents involved was assured throughout the conduct of the NHMS 2019.

Data Availability Statement: To protect the privacy of the respondents, the data set that supports the findings of this article is not publicly available. Request for data can be obtained from the Head of Centre for Biostatistics \& Data Repository, National Institutes of Health, Ministry of Health Malaysia on reasonable request and with the permission from the Director General of Health, Malaysia.

Acknowledgments: The authors would like to thank the Director General of Health, Malaysia for his permission to publish this article. We would like to thank all research team members for their contributions to this study. We are thankful for the kind cooperation of all respondents to the survey.

Conflicts of Interest: The author(s) declare that they have no conflicts of interest with respect to the research, authorship, and/or publication of this article.

\section{References}

1. Poortaghi, S.; Raiesifar, A.; Bozorgzad, P.; Golzari, S.E.J.; Parvizy, S.; Rafii, F. Evolutionary Concept Analysis of Health Seeking Behavior in Nursing: A Systematic Review. BMC Health Serv. Res. 2015, 15, 523. [CrossRef] [PubMed]

2. Olenja, J. Health Seeking Behaviour in Context. East Afr. Med. J. 2003, 80, 61-62. [CrossRef] [PubMed]

3. Chomi, E.N.; Mujinja, P.G.; Enemark, U.; Hansen, K.; Kiwara, A.D. Health Care Seeking Behaviour and Utilisation in a Multiple Health Insurance System: Does Insurance Affiliation Matter? Int. J. Equity Health 2014, 13, 25. [CrossRef] [PubMed]

4. Chan, C.Q.H.; Lee, K.H.; Low, L.L. A Systematic Review of Health Status, Health Seeking Behaviour and Healthcare Utilisation of Low Socioeconomic Status Populations in Urban Singapore. Int. J. Equity Health 2018, 17, 39. [CrossRef]

5. Lim, M.T.; Fong Lim, Y.M.; Tong, S.F.; Sivasampu, S. Age, Sex and Primary Care Setting Differences in Patients' Perception of Community Healthcare Seeking Behaviour towards Health Services. PLoS ONE 2019, 14, e0224260. [CrossRef]

6. Mok, C.Z.; Sellappans, R.; Loo, J.S.E. The Prevalence and Perception of Self-Medication among Adults in the Klang Valley, Malaysia. Int. J. Pharm. Pract. 2021, 29, 29-36. [CrossRef]

7. Mohamed Azhar, M.I.; Gunasekaran, K.; Kadirvelu, A.; Gurtu, S.; Sadasivan, S.; Kshatriya, B.M. Self-Medication: Awareness and Attitude among Malaysian Urban Population. Int. J. Collab. Res. Intern. Med. Public Health 2013, 5, 436-443.

8. Dawood, O.; Hassali, M.; Saleem, F.; Ibrahim, I.; Abdulameer, A.; Jasim, H. Assessment of Health Seeking Behaviour and Self-Medication among General Public in the State of Penang, Malaysia. J. Pharm. Pract. Res. 2017, 15, 991. [CrossRef] 
9. Pons, E.D.S.; Knauth, D.R.; Vigo, Á.; Mengue, S.S.; Gadelha, C.A.G.; Costa, K.S.; Do Nascimento, J.M.; Soeiro, O.M.; Mengue, S.S.; Da Motta, M.L.; et al. Predisposing Factors to the Practice of Self-Medication in Brazil: Results from the National Survey on Access, Use and Promotion of Rational Use of Medicines (PNAUM). PLoS ONE 2017, 12, e0189098. [CrossRef]

10. Ali, A. Self-Medication and Associated Health Care Costs- A Survey In The Urban And Rural Population of A Major City In Pakistan. Value Health 2015, 18, A171. [CrossRef]

11. Evans, R.G.; Stoddart, G.L. Producing Health, Consuming Health Care. Soc. Sci. Med. 1990, 31, 1347-1363. [CrossRef]

12. Jones, C.L.; Jensen, J.D.; Scherr, C.L.; Brown, N.R.; Christy, K.; Weaver, J. The Health Belief Model as an Explanatory Framework in Communication Research: Exploring Parallel, Serial, and Moderated Mediation. Health Commun. 2015, 30, 566-576. [CrossRef]

13. Grossman, M. On the Concept of Health Capital and the Demand for Health. J. Political Econ. 1972, 80, 223-255. [CrossRef]

14. Andersen, R.M. National Health Surveys and the Behavioral Model of Health Services Use. Med. Care 2008, 46, 647-653. [CrossRef]

15. Yahya, N.; Sukiman, N.K.; Suhaimi, N.A.; Azmi, N.A.; Manan, H.A. How Many Roads Must a Malaysian Walk down? Mapping the Accessibility of Radiotherapy Facilities in Malaysia. PLoS ONE 2019, 14, e0213583. [CrossRef]

16. Fujita, M.; Sato, Y.; Nagashima, K.; Takahashi, S.; Hata, A. Impact of Geographic Accessibility on Utilization of the Annual Health Check-Ups by Income Level in Japan: A Multilevel Analysis. PLoS ONE 2017, 12, e0177091. [CrossRef]

17. Falcon, D.J. The Health Care Gap in Rural Malaysia. New Malays. 2019, 37, 28-36.

18. Atun, R.; Berman, P.; Hsiao, W.; Myers, E.; Wei, A.Y. Malaysia Health Systems Research Volume 1: Contextual Analysis of the Malaysian Health System; Ministry of Health Malaysia; Harvard TH Chan School of Public Health: Putrajaya, Malaysia, 2016 ; pp. 35-164.

19. Department of Statistics Malaysia Labour Force Survey (LFS) Time Series Statistics by State, 1982-2019; Department of Statistics Malaysia: Putrajaya, Malaysia, 2020; pp. 1-32.

20. Ariff, K.M.; Teng, C.L. Rural Health Care in Malaysia. Aust. J. Rural Health 2002, 10, 99-103. [CrossRef]

21. Sivasampu, S.; Mohamad Noh, K.; Chin, M.C. Quality and Costs of Primary Care (QUALICOPC) Malaysia: Phase I-Public Clinics; Ministry of Health Malaysia ; Harvard TH Chan School of Public Health, Harvard University: Kuala Lumpur, Malaysia, 2015.

22. Andersen, R.M.; Davidson, P.L. Improving Access to Care in America: Individual and Contextual Indicators. In Changing the U.S Health Care System: Key Issues in Health Services Policy and Management; Jossey-Bass: San Francisco, CA, USA, 2007 ; pp. 3-31.

23. Levesque, J.F.; Harris, M.F.; Russell, G. Patient-Centred Access to Health Care: Conceptualising Access at the Interface of Health Systems and Populations. Int. J. Equity Health 2013, 12, 18. [CrossRef]

24. Lim, H.M.; Sivasampu, S.; Khoo, E.M.; Mohamad Noh, K. Chasm in Primary Care Provision in a Universal Health System: Findings from a Nationally Representative Survey of Health Facilities in Malaysia. PLoS ONE 2017, 12, e0172229. [CrossRef]

25. Haggerty, J.L.; Roberge, D.; Lévesque, J.F.; Gauthier, J.; Loignon, C. An Exploration of Rural-Urban Differences in HealthcareSeeking Trajectories: Implications for Measures of Accessibility. Health Place 2014, 28, 92-98. [CrossRef]

26. Andersen, R.M. Revisiting the Behavioral Model and Access to Medical Care: Does It Matter? J. Health Soc. Behav. 1995, 36, 1-10. [CrossRef]

27. Institute for Public Health (IPH) National Health and Morbidity Survey 2011 (NHMS 2011). In Healthcare Demand and Out-OfPocket Health Expenditure; Institute for Public Health: Kuala Lumpur, Malaysia, 2011; Volume 3, ISBN 9789673887682.

28. Institute for Public Health (IPH) \& Institute for Health Systems Research (IHSR) National Health and Morbidity Survey (NHMS) 2015. In Healthcare Demand; Institute for Public Health: Kuala Lumpur, Malaysia, 2015; Volume 3.

29. Mohd Rani, M.D.; Ithnin, M.; Mohamad Nor, A.U.; Juliana, N.; Mohd Effendy, N.; Azmani, S.; Abang Abdullah, K.H.; Mohd Aris, M.S.; Abu, I.F. Comparison of Health-Seeking Behaviour between Urban and Rural Malay Population of Negeri Sembilan, Malaysia. Int. J. Res. Pharm. Sci. 2019, 10, 3608-3615. [CrossRef]

30. Syed, S.T.; Gerber, B.S.; Sharp, L.K. Traveling towards Disease: Transportation Barriers to Health Care Access. J. Community Health 2013, 38, 976-993. [CrossRef]

31. Mat Lazim, M.S. Evolution of Migration for Urban and Rural; Department of Statistics Malaysia: Putrajaya, Malaysia, 2020; pp. 1-5.

32. Health Informatics Centre Planning and Development Division. Ministry of Health Malaysia. Health Facts 2020; Reference Data for year 2019; Health Informatics Centre: Putrajaya, Malaysia, 2020. Available online: https://www.moh.gov.my/moh/resources/ Penerbitan/PenerbitanUtama/HEALTHFACTS/HealthFacts2020.pdf (accessed on 5 December 2021).

33. Tew, M.M.; Hatah, E.; Arif, F.; Abdul Wahid, M.A.; Makmor-Bakry, M.; Abdul Maulad, K.N. Geospatial Analysis of Distribution of Community Pharmacies and Other Health Care Facilities Providing Minor Ailments Services in Malaysia. J. Pharm. Policy Pract. 2021, 14, 24. [CrossRef]

34. Jaafar, S.; Mohd Noh, K.; Abdul Muttalib, K.; Othman, N.H.; Healy, J.; Maskon, K.; Abdullah, A.R.; Zainuddin, J.; Abu Bakar, A.; Shaikh Abd Rahman, S.; et al. Malaysia Health System Review, 1st ed.; Healy, J., Ed.; Health Systems in Transition; World Health Organization: Geneva, Switzerland, 2013; Volume 3, ISBN 9789290615842.

35. Thomas, S.; Beh, L.; Nordin, R. bin Health Care Delivery in Malaysia: Changes, Challenges and Champions. J. Public Health Afr. 2011, 2, 93-97. [CrossRef]

36. Dhippayom, T.; Fuangchan, A.; Tunpichart, S.; Chaiyakunapruk, N. Opportunistic Screening and Health Promotion for Type 2 Diabetes: An Expanding Public Health Role for the Community Pharmacist. J. Public Health 2013, 35, 262-269. [CrossRef]

37. Phimarn, W.; Pianchana, P.; Limpikanchakovit, P.; Suranart, K.; Supapanichsakul, S.; Narkgoen, A.; Saramunee, K. Thai Community Pharmacist Involvement in Weight Management in Primary Care to Improve Patient's Outcomes. Int. J. Clin. Pharm. 2013, 35, 1208-1217. [CrossRef] 
38. Chua, S.S.; Lim, K.P.; Lee, H.G. Utilisation of Community Pharmacists by the General Public in Malaysia. Int. J. Pharm. Pract. 2013, 21, 66-69. [CrossRef]

39. Naeemmudeen, N.M.; Ghazali, N.A.N.M.; Bahari, H.; Ibrahim, R.; Samsudin, A.D.; Jasni, A.S. Trends in Antimicrobial Resistance in Malaysia. Med. J. Malays. 2021, 76, 698-705.

40. Llor, C.; Bjerrum, L. Antimicrobial Resistance: Risk Associated with Antibiotic Overuse and Initiatives to Reduce the Problem. Ther. Adv. Drug Saf. 2014, 5, 229-241. [CrossRef] [PubMed]

41. Ministry of Health Malaysia National Antimicrobial Guideline 2019, 3rd ed.; Pharmaceutical Services Programme, Ministry of Health Malaysia: Selangor, Malaysia, 2019; ISBN 9789675570780.

42. Ministry of Health Malaysia Malaysian Action Plan on Antimicrobial Resistance (MyAp-AMR) 2017-2021, 1st ed.; Ministry of Health Malaysia: Selangor, Malaysia, 2017; ISBN 978-967-2173-21-2.

43. Merican, M.I.; Bin Yon, R. Health Care Reform and Changes: The Malaysian Experience. Asia-Pac. J. Public Health/Asia-Pac. Acad. Consort. Public Health 2002, 14, 17-22. [CrossRef] [PubMed]

44. Institute for Health Systems Research (IHSR); National Institutes of Health. Ministry of Health Malaysia National Health and Morbidity Survey (NHMS) 2019. In Healthcare Demand; Institute for Health Systems Research: Selangor, Malaysia, 2020.

45. Government of Malaysia. Laws of Malaysia: Act 775 Traditional and Complementary Medicine Act 2016; National Printing Malaysia Berhad (Percetakan Nasional Malaysia Berhad): Kuala Lumpur, Malaysia, 2016. Available online: https://tcm.moh.gov.my/en/ upload/aktaBI2016.pdf (accessed on 5 December 2021).

46. Park, Y.L.; Canaway, R. Integrating Traditional and Complementary Medicine with National Healthcare Systems for Universal Health Coverage in Asia and the Western Pacific. Health Syst. Reform 2019, 5, 24-31. [CrossRef] [PubMed]

47. World Health Organization. The Regional Strategy for Traditional Medicine in the Western Pacific (2011-2020); World Health Organization Regional Office for the Western Pacific: Manila, Philiipines, 2012; ISBN 9789290615590.

48. Van der Hoeven, M.; Kruger, A.; Greeff, M.; Hausmann-Mueala, S.; Ribera, J.M.; Nyamongo, I.; Case, A.; Menendez, A.; Ardington, C.; Bradshaw, D.; et al. Differences in Health Care Seeking Behaviour between Rural and Urban Communities in South Africa. Int. J. Equity Health 2012, 11, 31. [CrossRef]

49. Masconi, K.L.; Matsha, T.E.; Echouffo-Tcheugui, J.B.; Erasmus, R.T.; Kengne, A.P. Reporting and Handling of Missing Data in Predictive Research for Prevalent Undiagnosed Type 2 Diabetes Mellitus: A Systematic Review. EPMA J. 2015, 6, 7. [CrossRef]

50. Chong, D.W.Q.; Jawahir, S.; Tan, E.H.; Sararaks, S. Redesigning a Healthcare Demand Questionnaire for National Population Survey: Experience of a Developing Country. Int. J. Environ. Res. Public Health 2021, 18, 4435. [CrossRef]

51. Jawahir, S.; Chong, D.W.Q.; Tan, E.H.; Mohd Noh, S.N.; CCY, F.; Kong, Y.L.; Halili, J. Revision of Healthcare Demand Questionnaire for National Health and Morbidity Survey (NHMS) 2019; Institute for Health Systems Research: Selangor, Malaysia, 2018.

52. Bursac, Z.; Gauss, C.H.; Williams, D.K.; Hosmer, D.W. Purposeful Selection of Variables in Logistic Regression. Source Code Biol. Med. 2008, 3, 17. [CrossRef]

53. Sultana, M.; Sarker, A.R.; Sheikh, N.; Akram, R.; Ali, N.; Mahumud, R.A.; Alam, N.H. Prevalence, Determinants and Health Care-Seeking Behavior of Childhood Acute Respiratory Tract Infections in Bangladesh. PLoS ONE 2019, 14, e0210433. [CrossRef]

54. Kong, Y.L.; Anis-Syakira, J.; Fun, W.H.; Balqis-Ali, N.Z.; Shakirah, M.S.; Sararaks, S. Socio-Economic Factors Related to Drinking Water Source and Sanitation in Malaysia. Int. J. Environ. Res. Public Health 2020, 17, 7933. [CrossRef]

55. Elnegaard, S.; Andersen, R.S.; Pedersen, A.F.; Larsen, P.V.; Søndergaard, J.; Rasmussen, S.; Balasubramaniam, K.; Svendsen, R.P.; Vedsted, P.; Jarbøl, D.E. Self-Reported Symptoms and Healthcare Seeking in the General Population -Exploring "the Symptom Iceberg. " BMC Public Health 2015, 15, 685. [CrossRef]

56. Zhang, Q.; Feng, S.; Wong, I.O.L.; Ip, D.K.M.; Cowling, B.J.; Lau, E.H.Y. A Population-Based Study on Healthcare-Seeking Behaviour of Persons with Symptoms of Respiratory and Gastrointestinal-Related Infections in Hong Kong. BMC Public Health 2020, 20, 402. [CrossRef]

57. Hassali, M.A.; Saleem, F.; Mustapa, M.D.; Bujang, C.P.; Mohd Ali, S.; Abu, S.F.; Abd Kadir, N.; Mohamad Yahaya, A.H.; Ahmad, K.; Siew, L.J.; et al. A National Survey on the Use of Medicines (NSUM) by Malaysian Consumers; Hassali, M.A., Saleem, F., Eds.; Pharmaceutical Services Division, Ministry of Health Malaysia: Selangor, Malaysia, 2016.

58. Dawkins, B.; Renwick, C.; Ensor, T.; Shinkins, B.; Jayne, D.; Meads, D. What Factors Affect Patients' Ability to Access Healthcare? An Overview of Systematic Reviews. Trop. Med. Int. Health 2021, 26, 1177-1188. [CrossRef]

59. Maung, T.M.; Deborah, S.; Narayanam, H.; Fernandez, K.; Kumari, U. Morbidity and Health Seeking Behavior among the Rural Population, Malaysia. Asian J. Med. Sci. 2020, 11, 1-5. [CrossRef]

60. Widayanti, A.W.; Green, J.A.; Heydon, S.; Norris, P. Health-Seeking Behavior of People in Indonesia: A Narrative Review. J. Epidemiol. Glob. Health 2020, 10, 6. [CrossRef]

61. Kuuire, V.Z.; Bisung, E.; Rishworth, A.; Dixon, J.; Luginaah, I. Health-Seeking Behaviour during Times of Illness: A Study among Adults in a Resource Poor Setting in Ghana. J. Public Health 2016, 38, e545-e553. [CrossRef]

62. Shahzad, M.; Upshur, R.; Donnelly, P.; Bharmal, A.; Wei, X.; Feng, P.; Brown, A.D. A Population-Based Approach to Integrated Healthcare Delivery: A Scoping Review of Clinical Care and Public Health Collaboration. BMC Public Health 2019, 19, 708. [CrossRef]

63. Lo Ying Ru, J.; Allotey, P. World Health Day 2018-Lessons from Malaysia on Universal Health Coverage. Available online: https:/ / www.who.int/malaysia/news/detail/18-04-2018-world-health-day-2018-\%E2\%80\%93-lessons-from-malaysiaon-universal-health-coverage (accessed on 5 December 2021). 
64. Rannan-Eliya, R.P.; Anuranga, C.; Manual, A.; Sararaks, S.; Jailani, A.S.; Hamid, A.J.; Razif, M.I.; Tan, E.H.; Darzi, A. Improving Health Care Coverage, Equity, and Financial Protection through a Hybrid System: Malaysia's Experience. Health Aff. 2016, 35, 838-846. [CrossRef]

65. Treleaven, E.; Whidden, C.; Cole, F.; Kayentao, K.; Traoré, M.B.; Diakité, D.; Sidibé, S.; Lin, T.K.; Boettiger, D.; Cissouma, S.; et al. Relationship between Symptoms, Barriers to Care and Healthcare Utilisation among Children under Five in Rural Mali. Trop. Med. Int. Health 2021, 26, 943-952. [CrossRef]

66. Oladipo, J.A. Utilization of Health Care Services in Rural and Urban Areas: A Determinant Factor in Planning and Managing Health Care Delivery Systems. Afr. Health Sci. 2014, 14, 322-333. [CrossRef]

67. Banerjee, S. Determinants of Rural-Urban Differential in Healthcare Utilization among the Elderly Population in India. BMC Public Health 2021, 21, 939. [CrossRef]

68. Fang, P.; Han, S.; Zhao, L.; Fang, Z.; Zhang, Y.; Zou, X. What Limits the Utilization of Health Services among the Rural Population in the Dabie Mountains- Evidence from Hubei Province, China? BMC Health Serv. Res. 2014, 14, 379. [CrossRef]

69. Shahar, S.; Vanoh, D.; Mat Ludin, A.F.; Ajit Singh, D.K.; Hamid, T.A. Factors Associated with Poor Socioeconomic Status among Malaysian Older Adults: An Analysis According to Urban and Rural Settings. BMC Public Health 2019, 19, 549. [CrossRef] [PubMed]

70. Jawahir, S.; Aziz, N. Self-Medication among Adult Population in Selangor, Malaysia. Int. J. Pharm. Pharm. Sci. 2017, 9, 268-274. [CrossRef]

71. Hassali, M.A.; Shafie, A.A.; Al-Qazaz, H.; Tambyappa, J.; Palaian, S.; Hariraj, V. Self-Medication Practices among Adult Population Attending Community Pharmacies in Malaysia: An Exploratory Study. Int. J. Clin. Pharm. 2011, 33, 794-799. [CrossRef]

72. Chua, S.S.; Sabki, N.H. Use of Nonprescription Medications by the General Public in the Klang Valley. J. Appl. Pharm. Sci. 2011, 1,93-98.

73. R Wijesinghe, P.; L Jayakody, R.; De A Seneviratne, R. Prevalence and Predictors of Self-Medication in a Selected Urban and Rural District of Sri Lanka. WHO South-East Asia J. Public Health 2012, 1, 28-41. [CrossRef]

74. Netherlands Enterprise Agency. Ministry of Foreign Affairs Malaysia Market Study Opportunities for the Dutch Life Sciences E Health Sector in Malaysia; Task Force Health Care: Hague, The Netherlands, 2019.

75. Karimy, M.; Rezaee-Momtaz, M.; Tavousi, M.; Montazeri, A.; Araban, M. Risk Factors Associated with Self-Medication among Women in Iran. BMC Public Health 2019, 19, 1033. [CrossRef]

76. Ocan, M.; Obuku, E.A.; Bwanga, F.; Akena, D.; Richard, S.; Ogwal-Okeng, J.; Obua, C. Household Antimicrobial Self-Medication: A Systematic Review and Meta-Analysis of the Burden, Risk Factors and Outcomes in Developing Countries. BMC Public Health 2015, 15, 742. [CrossRef]

77. Bennadi, D. Self-Medication: A Current Challenge. J. Basic Clin. Pharm. 2013, 5, 23. [CrossRef]

78. World Health Organization (WHO) and others Action on the Social Determinants of Health: Learning from Previous Experiences. In World 2010 Action; World Health Organization: Geneva, Switzerland, 2010.

79. Alghanim, S.A. Self-Medication Practice among Patients in a Public Health Care System. East. Mediterr. Health J. 2011, 17, 409-416. [CrossRef]

80. Sansgiry, S.S.; Bhansali, A.H.; Bapat, S.S.; Xu, Q. Abuse of Over-the-Counter Medicines: A Pharmacist's Perspective. Integr. Pharm. Res. Pract. 2017, 6, 6. [CrossRef]

81. Van der Heide, I.; Wang, J.; Droomers, M.; Spreeuwenberg, P.; Rademakers, J.; Uiters, E. The Relationship Between Health, Education, and Health Literacy: Results From the Dutch Adult Literacy and Life Skills Survey. J. Health Commun. 2013, 18, 172-184. [CrossRef] [PubMed]

82. Daim, N. 10-Year Wellness Plan to Guide Malaysia towards Healthy Living [NSTTV]. Available online: https:/ /www.nst.com. my/news/government-public-policy/2021/11/744442/10-year-wellness-plan-guide-malaysia-towards-healthy (accessed on 5 December 2021).

83. Isaac, V.; McLachlan, C.S.; Baune, B.T.; Huang, C.T.; Wu, C.Y. Poor Self-Rated Health Influences Hospital Service Use in Hospitalized Inpatients With Chronic Conditions in Taiwan. Medicine 2015, 94, e1477. [CrossRef] [PubMed]

84. Macassa, G.; Hiswåls, A.-S.; Ahmadi, N.; Alfredsson, J. Employment Status and Health Care Utilization in a Context of Economic Recession: Results of a Population Based Survey in East Central Sweden. Sci. J. Public Health 2014, 2, 610-616. [CrossRef]

85. Cislaghi, B.; Cislaghi, C. Self-Rated Health as a Valid Indicator for Health-Equity Analyses: Evidence from the Italian Health Interview Survey. BMC Public Health 2019, 19, 533. [CrossRef]

86. Zhang, Y.L.; Wu, B.J.; Chen, P.; Guo, Y. The Self-Rated Health Status and Key Influencing Factors in Middle-Aged and Elderly: Evidence from the CHARLS. Medicine 2021, 100, e27772. [CrossRef]

87. Li, X.; Deng, L.; Yang, H.; Wang, H. Effect of Socioeconomic Status on the Healthcare-Seeking Behavior of Migrant Workers in China. PLOS ONE 2020, 15, e0237867. [CrossRef]

88. Goncalves, J.; Martins, P.S. The Effect of Self-Employment on Health: Evidence from Longitudinal Social Security Data. IZA Discussion Paper No. 11305 2018. Available online: https:/ / docs.iza.org/dp11305.pdf (accessed on 5 December 2021).

89. Jabatan Perkhidmatan Awam Malaysia Pekeliling Perkhidmatan Bilangan 2 Tahun 2006: Pindaan Peraturan Berkaitan Penerimaan Sijil Sakit Swasta Untuk Tujuan Kemudahan Cuti Sakit; Jabatan Perkhidmatan Awam Malaysia: Kuala Lumpur, Malaysia, 2006; pp. 1-6.

90. Government of Malaysia. Malaysia Employment Act 1955. Available online: https://www.ilo.org/dyn/natlex/docs/WEBTEXT/ 48055/66265/E55mys01.htm (accessed on 5 December 2021). 
91. Rasul, F.B.; Kalmus, O.; Sarker, M.; Adib, H.I.; Hossain, M.S.; Hasan, M.Z.; Brenner, S.; Nazneen, S.; Islam, M.N.; De Allegri, M. Determinants of Health Seeking Behavior for Chronic Non-Communicable Diseases and Related out-of-Pocket Expenditure: Results from a Cross-Sectional Survey in Northern Bangladesh. J. Health Popul. Nutr. 2019, 38, 48. [CrossRef]

92. Mimi, O.; Tong, S.F.; Nordin, S.; Teng, C.L.; Khoo, E.M.; Abdul-Rahman, A.K.; Zailinawati, A.H.; Lee, V.K.M.; Chen, W.S.; Shihabudin, W.M.; et al. A Comparison of Morbidity Patterns in Public and Private Primary Care Clinics in Malaysia. Malays. Fam. Physician: Off. J. Acad. Fam. Physicians Malays. 2011, 6, 25.

93. Musinguzi, G.; Anthierens, S.; Nuwaha, F.; Van Geertruyden, J.P.; Wanyenze, R.K.; Bastiaens, H. Factors Influencing Compliance and Health Seeking Behaviour for Hypertension in Mukono and Buikwe in Uganda: A Qualitative Study. Int. J. Hypertens. 2018, 2018, 8307591. [CrossRef]

94. Mohamad Yunus, N.; Abd Manaf, N.H.; Omar, A.; Omar, M.A.; Salleh, M. Determinants of Healthcare Utilisation among the Elderly in Malaysia. Inst. Econ. 2017, 9, 115-140.

95. Zulkefli, Z.; Zaidi, M.A. Health Care Utilisation among the Elderly in Malaysia: Does Socioeconomic Status Matters? Persidang. Kebangs. Ekon. Malays. Ke VIII 2013, 3, 1141-1152.

96. Chan, C.M.H.; Ng, S.L.; In, S.; Wee, L.H.; Siau, C.S. Predictors of Psychological Distress and Mental Health Resource Utilization among Employees in Malaysia. Int. J. Environ. Res. Public Health 2021, 18, 314. [CrossRef]

97. United Nations Population Division Urban Population (\% of Total Population)—Malaysia. Available online: https://data. worldbank.org/indicator/SP.URB.TOTL.IN.ZS?locations=MY (accessed on 5 December 2021). 